\title{
Scalability of Atomic-Thin-Body (ATB) Transistors Based on Graphene Nanoribbons
}

\author{
Qin Zhang ${ }^{1}$, Yeqing Lu ${ }^{1}$, Huili Grace Xing ${ }^{1}$, Steven J. Koester ${ }^{2}$, and Siyuranga O. Koswatta ${ }^{3}$ \\ ${ }^{1}$ Department of Electrical Engineering, University of Notre Dame, Notre Dame, IN 46556, USA \\ Tel: (574) 631-5498, Fax: (574) 631-4393, email: qzhang1@nd.edu \\ ${ }^{2}$ University of Minnesota, 200 Union St. SE, Minneapolis, Twin Cities, MN 55455, USA \\ ${ }^{3}$ IBM Research Division, T. J. Watson Research Center, Yorktown Heights, NY 10598, USA
}

\begin{abstract}
A general solution for the electrostatic potential in an atomic-thin-body (ATB) field-effect transistor geometry is presented. The effective electrostatic scaling length, $\lambda_{\text {eff }}$, is extracted from the analytical model, which cannot be approximated by the lowest order eigenmode as traditionally done in SOI-MOSFETs. An empirical equation for the scaling length that depends on the geometry parameters is proposed. It is shown that even for a thick $\mathrm{SiO}_{2}$ back oxide $\lambda_{\text {eff }}$ can be improved efficiently by thinner top oxide thickness, and to some extent, with high-k dielectrics. The model is then applied to self-consistent simulation of graphene nanoribbon (GNR) Schottky-barrier field-effect transistors (SB-FETs) at the ballistic limit. In the case of GNR SB-FETs, for large $\lambda_{\text {eff, }}$, the scaling is limited by the conventional electrostatic short channel

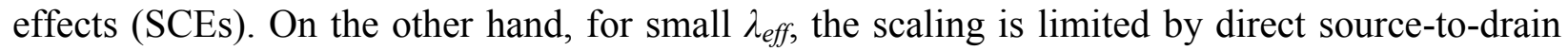
tunneling. A subthreshold swing below $100 \mathrm{mV} / \mathrm{dec}$ is still possible with a sub-10nm gate length in GNR SB-FETs.
\end{abstract}


Index Terms - Graphene, Schottky-barrier, thin-body, transistor scaling, subthreshold swing. 


\section{INTRODUCTION}

Graphene has been attracting considerable attention as an alternative channel material to silicon in CMOS technology [1]-[6] because of its remarkable electrical properties [1] as well as due to the fact that an energy band gap can be induced under certain conditions in this normally gapless material [2]-[4]. Therefore, it may be possible to realize atomic-thin-body (ATB) transistors based on graphene. To date, GNR based transistor operation has been numerically simulated using the nonequilibrium Green's function formalism combined with 3D electrostatics [5]-[6]. A generalized scaling theory for the ATB field-effect transistor (ATB-FET) geometry however, has not been presented, which is different from the well established ultrathin-body (UTB) structures which have comparatively large body thicknesses with a well-defined semiconductor dielectric medium [7]-[9]. In this letter, a new generalized analytical solution is derived to calculate the electrostatic potential in the ATB-FET geometry which is applicable to transistors based on single-layer and few-layer graphene as well as conventional semiconductor materials with body thicknesses below $\sim 1 \mathrm{~nm}[10]$. On the other hand, carrier transport properties will depend on the individual bandstructure of the specific material. Here, we simulate GNR based Schottky-barrier FETs (SB-FETs) to explore their scalability dependence on geometrical parameters.

\section{APPROACH}

The structure of a double-gate (DG) ATB transistor is illustrated in Fig. 1(a). The atomic thin channel material with a length $L_{g}$ and a negligible thickness (assumed to be zero in the model) is placed in between the top and back oxides. The top oxide has a thickness of $t_{t o x}$ and dielectric constant of $\varepsilon_{t o x}$, and the back oxide is $\mathrm{SiO}_{2}\left(\varepsilon_{b o x}=3.9 \varepsilon_{0}\right)$ with a thickness of $t_{b o x}$. The potential boundary conditions are as shown in Fig. 1(a). 
It should be noted that due to the negligible body thickness of ATB-FETs the parabolic approximation for the potential across the body cannot be used here as was done in original scaling studies [11]-[12]. Therefore, we use a procedure similar to that in [7]-[9], whereby the electrostatic potential of the ATB-FET can be written as $\psi(x, y)=v(x)+u l(x, y)+u r(x, y)$, where $v(x)$ is the long-channel solution that accounts for the top and bottom boundary conditions, as well as the self-consistent charge induced in the channel (therefore, the solution is valid in both subthreshold as well as above-threshold operation). $u l(x, y)$ and $u r(x, y)$ satisfy the source and drain boundary conditions, respectively, and can be expanded as:

$$
\begin{gathered}
u l(x, y)=\left\{\begin{array}{l}
\sum_{n=1}^{\infty} A_{n}^{l} \frac{\sin \left[k_{n}\left(x+t_{\text {tox }}\right)\right]}{\sin \left(k_{n} t_{\text {tox }}\right)} \frac{\sinh \left[k_{n}\left(L_{g}-y\right)\right]}{\sinh \left(k_{n} L_{g}\right)}\left(-t_{\text {tox }} \leq x \leq 0\right) \\
\sum_{n=1}^{\infty}-A_{n}^{l} \frac{\sin \left[k_{n}\left(x-t_{b o x}\right)\right]}{\sin \left(k_{n} t_{b o x}\right)} \frac{\sinh \left[k_{n}\left(L_{g}-y\right)\right]}{\sinh \left(k_{n} L_{g}\right)}\left(0 \leq x \leq t_{b o x}\right)
\end{array}\right. \\
u r(x, y)=\left\{\begin{array}{l}
\sum_{n=1}^{\infty} A_{n}^{r} \frac{\sin \left[k_{n}\left(x+t_{t o x}\right)\right]}{\sin \left(k_{n} t_{t o x}\right)} \frac{\sinh \left(k_{n} y\right)}{\sinh \left(k_{n} L_{g}\right)}\left(-t_{t o x} \leq x \leq 0\right) \\
\sum_{n=1}^{\infty}-A_{n}^{r} \frac{\sin \left[k_{n}\left(x-t_{b o x}\right)\right]}{\sin \left(k_{n} t_{b o x}\right)} \frac{\sinh \left(k_{n} y\right)}{\sinh \left(k_{n} L_{g}\right)}\left(0 \leq x \leq t_{b o x}\right)
\end{array}\right.
\end{gathered}
$$

where the eigenvalues $\lambda_{n}=1 / k_{n}$ are determined by the implicit equation, $\varepsilon_{\text {box }} \tan \left(k_{n} t_{t o x}\right)+\varepsilon_{\text {tox }} \tan \left(k_{n} t_{b o x}\right)=0$. The coefficients $A_{n}{ }^{l}$ and $A_{n}{ }^{r}$ are calculated by integrating $u l(x$, $0)^{*} g_{n}(x)$ and $\operatorname{ur}\left(x, L_{g}\right)^{*} g_{n}(x)$ from $-t_{t o x}$ to $t_{b o x}$, respectively, where $g_{n}(x)$ is constructed to be a set of corresponding conjugate functions to each term in $u l(x, 0)$ that satisfies $\int_{-t t o x}^{t b o x} u l_{n}(x, 0) g_{m}(x) d x=\delta_{n, m}$ (note that $g_{n}(x)$ will simultaneously satisfy a similar condition with each term of $u r\left(x, L_{g}\right)$ as well). The calculated eigenvalues $\lambda_{n}$ and coefficients $A_{n}{ }^{l}, A_{n}^{r}$ are shown in Fig. 1(b). Using the above series solution, an effective scaling length $\left(\lambda_{e f f}\right)$ is extracted by an exponential fitting of the potential profile near the source region. Since the eigenmodes are 
weighted by the expansion coefficients $A_{n}{ }^{l}$ and $A_{n}{ }^{r}$, which do not necessarily peak at $n=1$ (Fig. 1(b)), $\lambda_{\text {eff }}$ cannot be approximated by the first eigenvalue $\left(\lambda_{1}\right)$ as has been traditionally done in SOI-MOSFETs [7]-[9]. For the geometry considered in Fig. 1(b) the first eigenvalue gives $\lambda_{1} \approx$ $16 \mathrm{~nm}$, which shows a large discrepancy to the $2 \mathrm{D}$ series solution with $\lambda_{\text {eff }} \approx 1.6 \mathrm{~nm}$.

We use the above series solution to simulate GNR SB-FETs, considering a 2nm-wide GNR with an energy band gap of $E_{g}=0.69 \mathrm{eV}$ [13], and source/drain SB height of $\Phi_{B}=0.1 \mathrm{~V}$. The Schottky barrier and direct source-to-drain $(S \rightarrow D)$ tunneling are both included on the same footing, whereby the WKB tunneling coefficient is calculated using the imaginary bandstructure of the GNR that treats the electron-hole nature of carriers realistically. Ballistic transport simulations are performed similar to [14] accounting for self-consistent electrostatics, while any edge-induced states in the band gap [15] and phonon scattering [16] are not considered.

\section{RESULTS AND DISCUSSION}

Figure 2(a) compares the extracted $\lambda_{\text {eff }}$ to the lowest order eigenvalue $\lambda_{1}$ as a function of $t_{\text {tox }}$ for different $t_{b o x}$ values. It is clearly seen that $\lambda_{1}$ cannot correctly describe $\lambda_{\text {eff. }}$ Therefore, an empirical equation $\lambda_{e m p}=\alpha t_{t o x}\left[1+\frac{\varepsilon_{b o x}}{\varepsilon_{t o x}}\right]\left[1+\left(\frac{\varepsilon_{b o x}}{\varepsilon_{t o x}}\right)\left(\frac{t_{t o x}}{t_{b o x}}\right)\right] /\left[1+\frac{t_{t o x}}{t_{b o x}}\right]$ is proposed to approximate $\lambda_{\text {eff }}$, and this equation shows reasonable consistency for a large range of $t_{t o x}$ and $\varepsilon_{t o x}$ values at thick $\mathrm{SiO}_{2}$ box limit where $\alpha=0.6$ is the only fitting parameter (Fig. 2). It is seen in Fig. 2(a) that $\lambda_{\text {eff }}$ is greatly improved by thinner $t_{t o x}$, and also modulated by $t_{b o x}$ when it is below a certain thickness $(<50 \mathrm{~nm})$. On the other hand, $\lambda_{\text {eff }}$ and $\lambda_{\text {emp }}$ do not appreciably depend on $t_{\text {box }}$ for thicknesses $>50 \mathrm{~nm}$. Figure 2(b) plots $\lambda_{\text {eff }}$ as a function of $\varepsilon_{t o x}$ for different $t_{t o x}$, and shows that $\lambda_{\text {eff }}$ is also improved by high-k top oxide, but the improvement saturates at a certain point [5] [7]. More importantly, even for a thick $\mathrm{SiO}_{2}$ back oxide, $\lambda_{\text {eff }}$ can be reduced below $2 \mathrm{~nm}$ by thinning the top oxide thickness. 
The transfer characteristics of GNR SB-FETs are shown in Fig. 3(a) for different gate lengths. For the calculated geometry, $I_{o n} / I_{\text {off }}>10^{3}$ is achieved at a supply voltage of $0.3 \mathrm{~V}$. Figures 3(b) and 3(c) show the band diagram and the energy-resolved current density in the offstate of the $L_{g}=10 \mathrm{~nm}$ and $15 \mathrm{~nm}$ devices, respectively. For this geometry which has $\lambda_{\text {eff }}=$ $1.45 \mathrm{~nm}$, when the channel length is $L_{g} \geq 15 \mathrm{~nm}$ the subthreshold swing (SS) approaches the $60 \mathrm{mV} / \mathrm{dec}$ limit, but it degrades to $85 \mathrm{mV} / \mathrm{dec}$ at $L_{g}=10 \mathrm{~nm}$ because of direct $\mathrm{S} \rightarrow \mathrm{D}$ tunneling (Fig. 3(b)). Here, we extract the $S S$ values where $\log _{10}\left(I_{D}\right)-V_{G S}$ is nearly linear in Fig. 3(a) at $V_{G S}$ $\approx 0.05 \mathrm{~V}$. In order to further elucidate the scaling potential of GNR SB-FETs, Fig. 4(a) plots $S S$ vs. $L_{g}$, and Fig. 4 (b) plots $S S$ vs. $L_{g} /\left(\pi \lambda_{e f f}\right)$. Since the $2 \mathrm{D}$ potential at the middle of the channel near the thermionic barrier region depends on $\exp \left(-L_{g} / 2 . \lambda_{\text {eff }}\right)$, previous works [7] [9] [12] have shown that $S S$ degrades significantly for $L_{g} /\left(\pi \lambda_{\text {eff }}\right) \leq 1.5 \sim 2$ due to excessive thermal leakage.

We, however, identify two distinct limiting conditions for $S S$ degradation depending on the device geometrical parameters that are yet practically relevant: 1) for very small $\lambda_{\text {eff }}$ values, $S S$ degradation is mainly due to direct $\mathrm{S} \rightarrow \mathrm{D}$ tunneling, while 2) at larger $\lambda_{\text {eff, }} S S$ degradation is due to conventional electrostatic short-channel-effects (SCEs) [7] [9] [12]. For thin $t_{t o x}$ (1nm and $2.15 \mathrm{~nm}$ ) in Fig. 4(a) with small $\lambda_{\text {eff }}, S S$ degradation for $L_{g}$ below $\sim 15 \mathrm{~nm}$ is due to direct $\mathrm{S} \rightarrow \mathrm{D}$ tunneling [5], and not because of electrostatic SCEs. An $S S \leq 100 \mathrm{mV} / \mathrm{dec}$ is still possible for sub$10 \mathrm{~nm} L_{g}$ with small $\lambda_{\text {eff }}$ values, even though obtaining small off-state currents will be a challenge because of the direct tunneling limit. Nevertheless, narrower GNRs with larger band gaps may alleviate this problem [5]. For thick $t_{t o x}(5 \mathrm{~nm}$ and $8 \mathrm{~nm})$ in Fig. 4(b), on the other hand, with large $\lambda_{\text {eff, }} S S$ increases to $\sim 100 \mathrm{mV} / \mathrm{dec}$ when the conventional $L_{g} /\left(\pi \lambda_{\text {eff }}\right) \approx 1.5$ condition is met [7] [9] [12]. In other words, in the large $\lambda_{\text {eff }}$ limit, scaling is controlled by the electrostatic SCEs. Interestingly, when comparing Fig. 2 with SOI-MOSFET simulations in [9] for a similar device 
geometry, the GNR channel has smaller $\lambda_{\text {eff }}$ than the $5 \mathrm{~nm}$ thick Si based UTB channel, showing the excellent scaling potential of GNR based ATB-FETs.

\section{CONCLUSIONS}

A generalized 2D analytical electrostatic solution is developed for the ATB-transistor geometry. An empirical equation for the effective scaling length has been proposed which cannot be approximated by the lowest order eigenmode as traditionally done in SOI-MOSFETs. Even for a thick $\mathrm{SiO}_{2}$ back oxide, the scaling length can be efficiently improved by thinner top oxide, and to some extent, by high-k dielectrics. It is seen that the scalability of GNR SB-FETs can be limited either by direct $\mathrm{S} \rightarrow \mathrm{D}$ tunneling, or conventional electrostatic SCEs, depending on the geometrical parameters. Compared to conventional UTB-FET geometries, excellent scaling potential of ATB-FETs based on graphene has been demonstrated. 


\section{REFERENCES}

[1] A. K. Geim, "Graphene: status and prospects," Science, vol. 324, pp.1530-1534, 2009.

[2] M. C. Lemme, T. J. Echtermeyer, M. Baus, and H. Kurz, "A graphene field-effect device," IEEE Electron Dev. Lett., vol. 28, no. 4, pp. 282-284, 2007.

[3] Z. Chen, Y. -M. Lin, M. J. Rooks, and P. Avouris, "Graphene nano-ribbon electronics," Physica E, vol. 40, pp. 228-232, 2007.

[4] E. McCann, "Asymmetry gap in the electronic band structure of bilayer graphene," Phys. Rev. B, vol. 74, no. 16, p. 161403, 2006.

[5] Y. Ouyang, Y. Yoon, and J. Guo, "Scaling behaviors of graphene nanoribbon FETs: a three-dimensional quantum simulation study," IEEE Trans. Electron Dev., vol. 54, no. 9, pp. 2223-2231, 2007.

[6] G. Fiori and G. Iannaccone, "Simulation of graphene nanoribbon field-effect transistors," IEEE Electron Dev. Lett., vol. 28, no.8, pp. 760-762, 2007.

[7] D. J. Frank, Y. Taur, and H. -S. P. Wong, "Generalized scale length for two-dimensional effects in MOSFETs," IEEE Electron Dev. Lett., vol. 19, no. 10, pp. 385-387, 1998.

[8] S. -H. Oh, D. Monroe, J. M. Hergenrother, "Analytic description of short-channel effects in fully depleted double-gate and cylindrical, surrounding-gate MOSFETs," IEEE Electron Dev. Lett., vol. 21, no. 9, pp. 445-447, 2000.

[9] X. Liang and Y. Taur, "A 2-D analytical solution for SCEs in DG MOSFETs," IEEE Trans. Electron Dev., vol. 51, no. 8, pp. 1385-1391, 2004.

[10] M. Schmidt, M. C. Lemme, H. D. B. Gottlob, F. Driussi, L. Selmi, and H. Kurz, "Mobility extraction in SOI MOSFETs with sub $1 \mathrm{~nm}$ body thickness," Solid-State Electronics, vol. 53, no. 12, pp. 1246-1253, 2009. 
[11] K. K. Young, "Short-channel effect in fully depleted SOI MOSFETs," IEEE Trans. Electron Dev., vol. 36, no. 2, pp. 399-402, 1989.

[12] R. H. Yan, A. Ourmazd, and K. F. Lee, "Scaling the Si MOSFET - from bulk to SOI to bulk," IEEE Trans.Electron Dev., vol. 39, no. 11, pp. 1704-1710, 1992.

[13] B. Trauzettel, D. V. Bulaev, D. Loss, and G. Burkard, "Spin qubits in graphene quantum dots," Nature Phys., vol. 3, pp. 192-196, 2007.

[14] L. C. Castro, D. L. John and D. L. Pulfrey, "Towards a compact model for schottkybarrier nanotube FETs," COMMAD2002, pp. 303-306.

[15] D. Basu, M. J. Gilbert, L. F. Register, S. K. Banerjee, A. H. MacDonald, "Effect of edge roughness on electronic transport in graphene nanoribbon channel metal-oxidesemiconductor field-effect transistors," Appl. Phys. Lett., vol. 92, no. 4, p. 042114, 2008.

[16] S. O. Koswatta, M. S. Lundstrom, and D. E. Nikonov, "Influence of phonon scattering on the performance of p-i-n band-to-band tunneling transistors," Appl. Phys. Lett., vol. 92, p. $043125,2008$. 


\section{FIGURE CAPTIONS}

Fig. 1: (a) Schematic cross section of a double-gate (DG) ATB transistor. The channel material is buried between the top and the back oxides with zero thickness. (b) Eigenvalues $\lambda_{n}$ and coefficients $A_{n}{ }^{l}$ and $A_{n}{ }^{r}$ in the potential expansion (Eq. (1) and (2)) of a DG GNR SB-FET with $t_{b o x}=50 \mathrm{~nm}\left(\mathrm{SiO}_{2}\right), t_{t o x}=2.15 \mathrm{~nm}\left(\right.$ high-k with $\left.\varepsilon_{t o x}=20 \varepsilon_{0}\right)$, and $V_{G S}=V_{D S}$ $=0.3 \mathrm{~V}$.

Fig. 2: (a) The extracted effective scaling length $\lambda_{\text {eff, }}$, the lowest order eigenvalue $\lambda_{1}$ and the empirical $\lambda_{e m p}$ as a function of $t_{t o x}$ for different $t_{b o x}$. The back oxide is $\mathrm{SiO}_{2}$ and the top oxide is high-k with $\varepsilon_{t o x}=20 \varepsilon_{0}$. (b) $\lambda_{\text {eff }}$ and $\lambda_{\text {emp }}$ as a function of $\varepsilon_{\text {tox }}$ for different $t_{t o x}$. The back oxide is $50 \mathrm{~nm} \mathrm{SiO}_{2}$.

Fig. 3: (a) Calculated transfer characteristics of DG GNR SB-FETs with different gate lengths at $\lambda_{\text {eff }}=1.45 \mathrm{~nm}$. Band diagram and current density per energy in the off-state for a DG GNR SB-FET with (b) $L_{g}=10 \mathrm{~nm}$ and (c) $L_{g}=15 \mathrm{~nm}$, showing that the off-state leakage is dominated by thermionic current at longer channel length (c), and increased dramatically by direct source-to-drain tunneling at short gate length (b) for this geometry $\left(\lambda_{\text {eff }}=1.45 \mathrm{~nm}\right)$.

Fig. 4: The subthreshold swing (SS) vs. (a) $L_{g}$, and (b) $L_{g} / \lambda_{\text {eff. For }}$ thin $t_{\text {tox }}$ (small $\lambda_{\text {eff }}$ ), $S S$ significantly increases for $L_{g}$ below $15 \mathrm{~nm}$ due to direct source-to-drain tunneling, while for thick $t_{\text {tox }}$ (large $\left.\lambda_{\text {eff }}\right), S S$ increases to $100 \mathrm{mV} /$ dec when $L_{g} /\left(\pi \lambda_{\text {eff }}\right) \approx 1.5$ due to electrostatic short-channel-effects (SCEs), as observed in conventional SOI-MOSFETs [7] [9] [12]. 


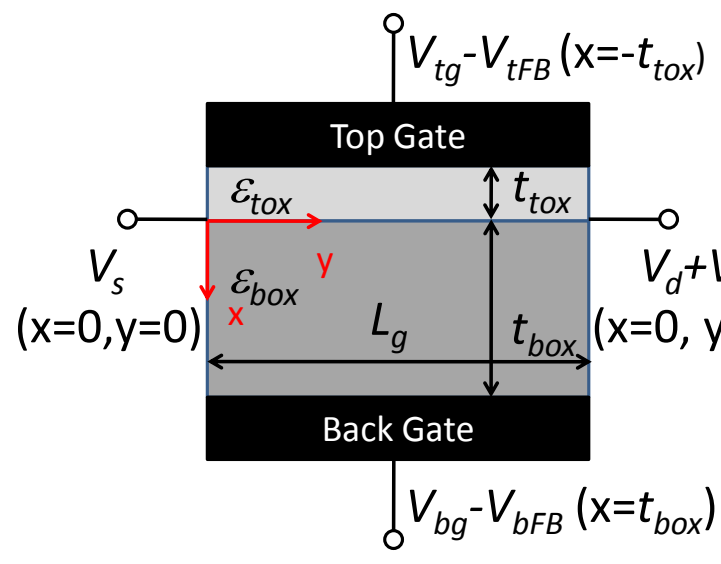

(a)

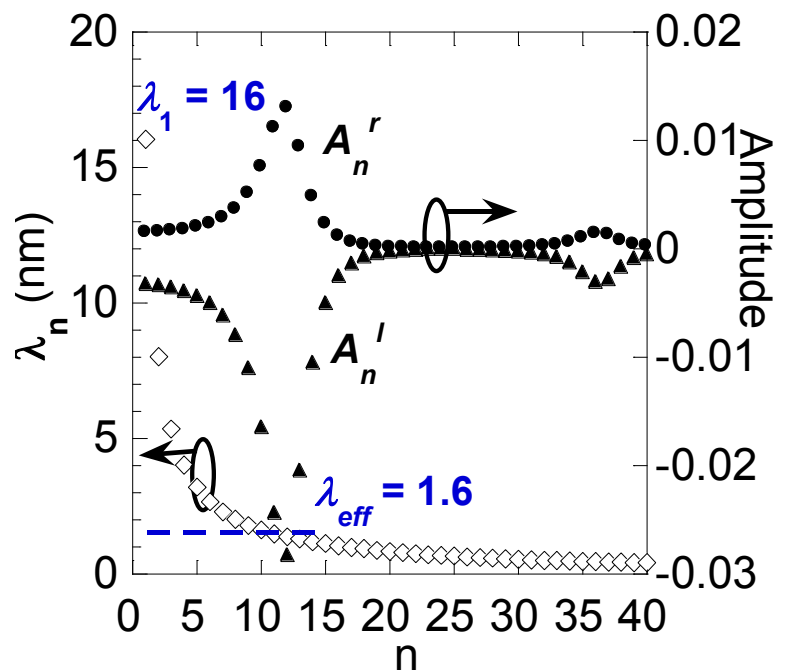

(b)

Fig. 1. Q. Zhang, et al., IEEE Elect. Dev. Lett. 

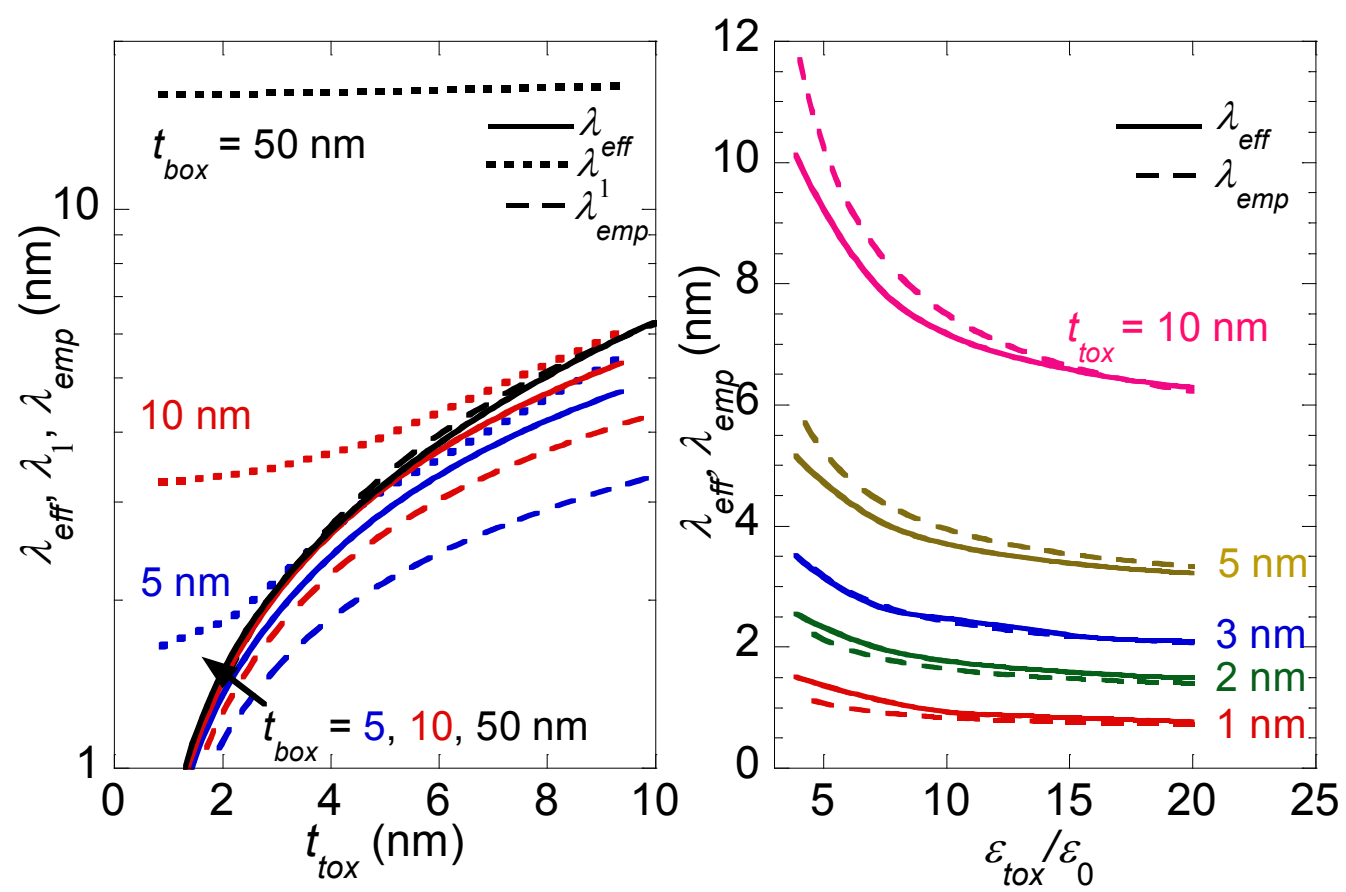

Fig. 2. Q. Zhang, et al., IEEE Elect. Dev. Lett. 


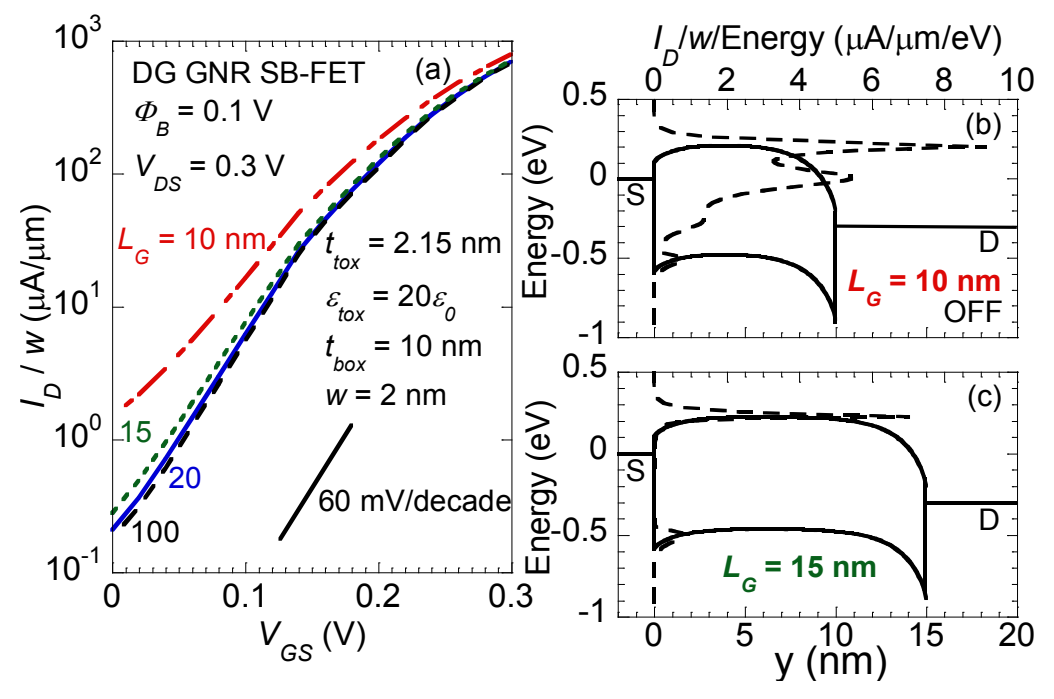

Fig. 3. Q. Zhang, et al., IEEE Elect. Dev. Lett. 


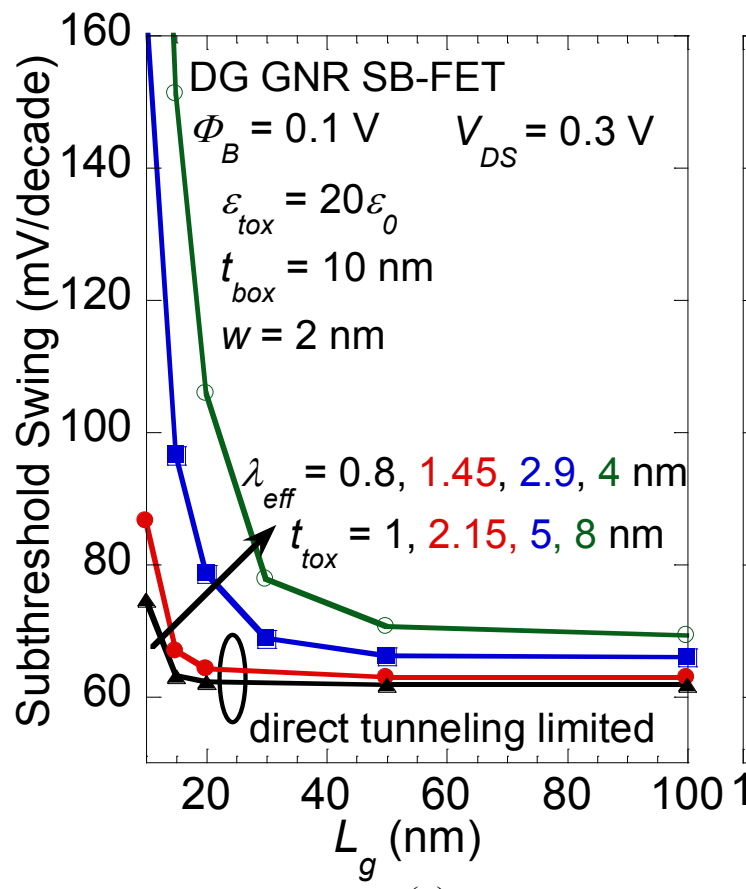

(a)

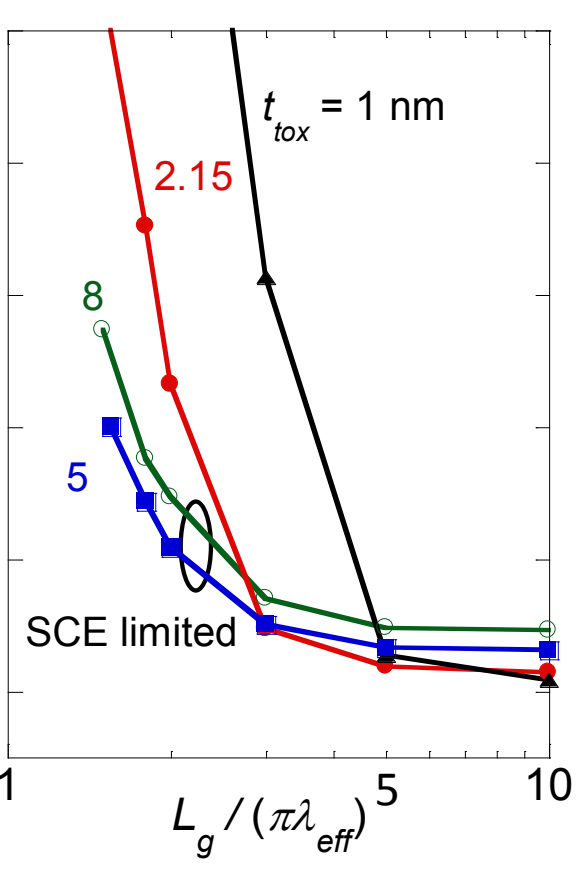

(b)

Fig. 4. Q. Zhang, et al., IEEE Elect. Dev. Lett. 